Short Communication

\title{
On the optimality of the window method in computational homogenization
}

\author{
I. Temizer ${ }^{\mathrm{a}, *}, \mathrm{~T} . \mathrm{Wu}^{\mathrm{b}}$, P. Wriggers ${ }^{\mathrm{b}}$ \\ a Department of Mechanical Engineering, Bilkent University, 06800 Ankara, Turkey \\ ${ }^{\mathrm{b}}$ Institute of Continuum Mechanics, Leibniz University of Hannover, Appelstr. 11, 30167 Hannover, Germany
}

\section{A R T I C L E I N F O}

\section{Article history:}

Received 8 October 2012

Received in revised form 11 December 2012

Accepted 24 December 2012

Available online 30 January 2013

\section{Keywords:}

Computational homogenization

Self-consistent scheme

Thermal conduction

\begin{abstract}
A B S T R A C T
The window method, where the microstructural sample is embedded into a frame of a homogeneous material, offers an alternative to classical boundary conditions in computational homogenization. Experience with the window method, which is essentially the selfconsistent scheme but with a finite surrounding medium instead of an infinite one, indicates that it delivers faster convergence of the macroscopic response with respect to boundary conditions of pure essential or natural type as the microstructural sample size is increased to ensure statistical representativeness. In this work, the variational background for this observed optimal convergence behavior of the homogenization results with the window method is provided and the method is compared with periodic boundary conditions that it closely resembles.
\end{abstract}

(c) 2013 Elsevier Ltd. All rights reserved.

\section{Introduction}

Boundary conditions (BCs) play an important role in homogenization. In the context of mechanical problems, they are primarily of three types: (i) pure displacement, (ii) pure traction, and (iii) periodic. Periodic BCs are indispensable in the mathematical theory of homogenization (Pavliotis \& Stuart, 2008) whereas the former two are of particular significance in analytical approaches for random microstructures and are typically combined with self-consistency schemes (NematNasser \& Hori, 1999). Computational homogenization techniques, which often build upon and improve existing analytical methods, may be more favorable to pursue if exact or sufficiently accurate microstructural data is available (Zohdi \& Wriggers, 2005). Within such methods, one clearly observes the effect of the employed BCs on various macroscopic quantities of interest in a wide range of physical problems (Ostoja-Starzewski, 2006). A basis for these observations can be robustly stated in linearized problems. In the context of mechanical problems, an ordering of the macroscopic elastic properties obtained under pure displacement and pure traction BCs was proven in Huet (1990), later extended to include BCs of the periodic type in Hazanov and Huet (1994). It was additionally demonstrated that BC effects could be profitably incorporated into a scheme whereby arbitrarily refinable computational bounds are constructed (Huet, 1990; Zohdi, 2002), in the spirit of refinable higher-order analytical bounds (Torquato, 2002).

There are, however, cases where a direct application of the these BCs may be unfavorable. For instance, when soft phases or voids are present in the vicinity of the boundary, pure traction and periodic type BCs may cause overly deformed meshes that are numerically undesirable (Miehe $\&$ Koch, 2002). In fact, these BCs are simply not applicable when voids intersect the boundary of the analysis domain. This leaves pure displacement type BCs as an option. These, on the other hand, can significantly overestimate the macroscopic stiffness for a given microstructural sample (Zohdi \& Wriggers, 2005). A remedy to these prob-

\footnotetext{
* Corresponding author. Tel.: +90 (312) 2903064.

E-mail address: temizer@bilkent.edu.tr (İ. Temizer).
} 
lems is to embed the sample into a frame of a homogeneous material following similar ideas from analytical approaches. This window method was proposed in Babuška, Andersson, Smith, and Levin (1999) where the window frame material was chosen as the matrix. Average stresses and strains were monitored in the entire extended domain and hence a correction factor was introduced in order to alleviate the effect of the frame. The idea was later employed in Hain and Wriggers (2008) where the frame material was represented by the average stiffness of the microstructural constituents while still monitoring average quantities in the sample only. Finally, this approach was further developed towards a self-consistent scheme in Krabbenhøft, Hain, and Wriggers (2008) wherein the frame material was iteratively updated until it matched the macroscopic response obtained from the sample. See also Düster, Sehlhorst, and Rank (2012) for an application to three-dimensional cellular microstructures as well as Zohdi (2010) where the frame material is representative of the original heterogeneous medium.

Experience with the window method indicates that it delivers faster convergence of the macroscopic response with respect to BCs of pure displacement or traction type as the microstructural sample size is increased, a procedure that is necessary to assess the statistical representativeness of the sample. In this work, the variational background for this observed optimal convergence behavior of the homogenization results with the window method is provided. The emphasis is on the effects of employing frames with finite width since the case of an infinite surrounding frame medium corresponds to the classical self-consistent scheme. A detailed overview of self-consistent methods is given in Kanaun and Levi (2008) and a recent discussion of their generalizations in Benveniste (2008). These methods have classically been applied to particulateor fiber-reinforced composites, although extensions to more complicated heterogeneous media such as polycrystals have also been pursued (Jiang \& Weng, 2004; Su \& Weng, 2006). Presently, no restrictions are imposed on the microstructural geometry however the presentation is limited to linear thermal conduction. The same variational principles apply to other physical phenomena within a linear framework in a straightforward fashion, in particular to diffusion or elasticity. An extension to the analysis of the overall electromagnetic response of heterogeneous media does not follow from this presentation yet is expected to be realizable with additional effort in view of the applicability of classical micromechanics techniques to this physical regime (Benveniste \& Milton, 2011; Su \& Weng, 2006; Zohdi, 2010).

\section{Variational framework}

\subsection{Minimum principles}

As the classical homogenization problem posed on a microstructural sample $\mathcal{V}$, with boundary $\partial \mathcal{V}=\partial \mathcal{V}^{\theta} \cup \partial \mathcal{V}^{h}$, the steady-state energy balance is considered in the absence of an external heat supply. The problem may be stated within a variational setting via the functional

$$
\mathcal{D}(\theta)=\int_{\mathcal{V}} \frac{1}{2} \mathbf{g} \cdot \boldsymbol{K g} \mathrm{d} v-\int_{\partial \mathcal{V}^{h}} \theta \bar{h} \mathrm{~d} a .
$$

Here, $\theta$ is the temperature, $\boldsymbol{g}=\operatorname{grad}[\theta]$ and $\boldsymbol{K}$ is the thermal conductivity tensor. The corresponding heat flux is $\boldsymbol{q}=-\boldsymbol{K g}$. The solution $\theta$ to the problem among all admissible temperature fields -those which satisfy the essential BCs $\theta=\bar{\theta}$ on $\partial \mathcal{V}^{\theta}-$ minimizes $\mathcal{D}$, implying the energy balance $\operatorname{div}[\boldsymbol{q}]=0$ in $\mathcal{V}$ as well as the natural BCs $h=\bar{h}$ on $\partial \mathcal{V}^{h}$. Here, $h=-\boldsymbol{q} \cdot \boldsymbol{n}$ is the normal heat flux on a surface having outward unit normal $\boldsymbol{n}$. The same problem may also be stated within a complementary setting. Consider

$$
\mathcal{D}^{c}(\mathbf{q})=\int_{\mathcal{V}} \frac{1}{2} \boldsymbol{q} \cdot \boldsymbol{R} \boldsymbol{q} \mathrm{d} v-\int_{\partial \mathcal{V}^{\theta}} \bar{\theta} h \mathrm{~d} a,
$$

where $\boldsymbol{R}=\boldsymbol{K}^{-1}$ is the thermal resistivity tensor. The solution $\boldsymbol{q}$ to the problem among all admissible flux fields - those which satisfy $\operatorname{div}[\boldsymbol{q}]=0$ in $\mathcal{V}$ and $h=\bar{h}$ on $\partial \mathcal{V}^{h}$-is the one that minimizes $\mathcal{D}^{c}$, implying $\theta=\bar{\theta}$ on $\partial \mathcal{V}^{\theta}$.

\subsection{Window method}

The variational results apply to any domain $\boldsymbol{\Omega}$. Typically, linear (LN), uniform (UF) or periodic (PR) BCs on $\partial \boldsymbol{\Omega}$ are of interest:

$$
\begin{aligned}
& \text { LT-BCs }: \theta=\overline{\mathbf{g}} \cdot \boldsymbol{x}, \quad \text { UF-BCs }: h=-\overline{\mathbf{q}} \cdot \boldsymbol{n}, \\
& \text { PR-BCs }: \theta^{+}-\theta^{-}=\overline{\mathbf{g}} \cdot\left(\boldsymbol{x}^{+}-\boldsymbol{x}^{-}\right) \text {and } h^{+}=-h^{-} .
\end{aligned}
$$

With PR-BCs, the boundary of the domain is partitioned into periodically linked + and - regions. The notation

$$
\langle\mathcal{Q}\rangle_{\Omega}=\frac{1}{|\Omega|} \int_{\Omega} \mathcal{Q} \mathrm{d} v
$$

for the average of an arbitrary quantity $\mathcal{Q}$ will be useful for compactness. Standard averaging theorems (Nemat-Nasser $\&$ Hori, 1999) are then recalled under the assumptions of a diverge-free flux field and a continuous temperature field. The theorems state that for LT- and PR-BCs on $\partial \Omega$ one obtains $\langle\mathbf{g}\rangle_{\Omega}=\overline{\mathbf{g}}$ and for UF-BCs $\langle\boldsymbol{q}\rangle_{\Omega}=\overline{\mathbf{q}}$. Additionally, for all the BCs in (2.3),

$$
\langle\mathbf{q} \cdot \mathbf{g}\rangle_{\Omega}=\langle\mathbf{q}\rangle_{\Omega} \cdot\langle\mathbf{g}\rangle_{\Omega}
$$




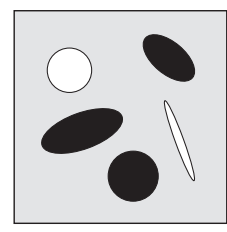

$\mathcal{V}$

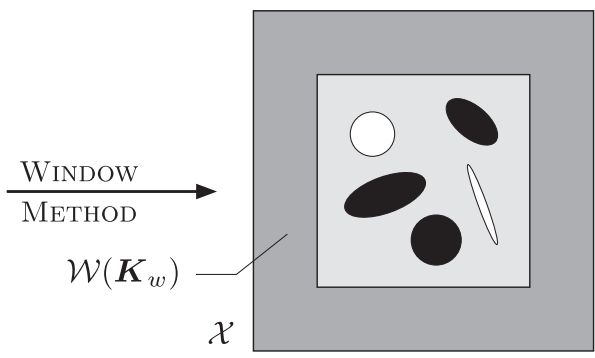

Fig. 1. The window method is summarized

The microstructure is now embedded within a homogeneous frame $\mathcal{W}$ and the extended domain $\mathcal{V} \cup \mathcal{W}$ is denoted with $\mathcal{X}$ (Fig. 1). The frame is assigned a judiciously chosen material property $\boldsymbol{K}_{w}=\boldsymbol{R}_{w}^{-1}$. After solving the problem, subject to a set of BCs in the form above, the homogenized response is characterized through the macroscopic tensors $\overline{\boldsymbol{K}}=\overline{\boldsymbol{R}}^{-1}$ such that

$$
\langle\mathbf{q}\rangle_{\mathcal{V}}=-\overline{\mathbf{K}}\langle\mathbf{g}\rangle_{\mathcal{V}}
$$

However, the window method is incomplete in this form. It is additionally required that the solution from the window method satisfies self-consistency-replacing $\boldsymbol{K}_{w}$ by $\overline{\boldsymbol{K}}$ followed by a repetition of the solution should again deliver $\overline{\boldsymbol{K}}$. In practice, self-consistency is satisfied within a few iterations to very good accuracy, as to be demonstrated.

It is important to highlight that the domain of averaging in (2.6) is the microstructure only. Note that (Zohdi \& Wriggers, 2005)

$$
\langle\mathcal{Q}\rangle_{\mathcal{X}}=f_{v}\langle\mathcal{Q}\rangle_{\mathcal{V}}+f_{w}\langle\mathcal{Q}\rangle_{\mathcal{W}}
$$

where $f_{v}=|\mathcal{V}| /|\mathcal{X}|$ and $f_{w}=|\mathcal{W}| /|\mathcal{X}|=1-f_{v}$. Therefore, without self-consistency,

$$
\langle\boldsymbol{q}\rangle_{\mathcal{X}}=f_{v}\langle\boldsymbol{q}\rangle_{\mathcal{V}}+f_{w}\langle\boldsymbol{q}\rangle_{\mathcal{W}}=-f_{v} \overline{\mathbf{K}}\langle\mathbf{g}\rangle_{\mathcal{V}}-f_{w} \boldsymbol{K}_{w}\langle\mathbf{g}\rangle_{\mathcal{W}}=-\overline{\mathbf{K}}\left(f_{v}\langle\mathbf{g}\rangle_{\mathcal{V}}+f_{w} \overline{\boldsymbol{K}}^{-1} \boldsymbol{K}_{w}\langle\mathbf{g}\rangle_{\mathcal{W}}\right) \neq-\overline{\mathbf{K}}\langle\mathbf{g}\rangle_{\mathcal{X}}
$$

Only at convergence of the iterations is $\boldsymbol{K}_{w}=\overline{\boldsymbol{K}}$ so that the domain of averaging can be $\mathcal{V}$ or $\mathcal{X}$.

\section{Augmented ordering relationship}

Let $\left\{\overline{\boldsymbol{K}}_{\mathrm{LT}}, \overline{\boldsymbol{K}}_{\mathrm{UF}}, \overline{\boldsymbol{K}}_{\mathrm{PR}}\right\}$ denote the homogenized conductivities under different BCs obtained using the window method. Without the frame, it is well-known (Hazanov \& Huet, 1994) that the ordering relationship

$$
\overline{\boldsymbol{K}}_{\mathrm{UF}}^{(0)} \leqslant \overline{\boldsymbol{K}}_{\mathrm{PR}}^{(0)} \leqslant \overline{\boldsymbol{K}}_{\mathrm{LT}}^{(0)}
$$

holds, where the superscript is used to denote that there is no frame and $\boldsymbol{A} \leqslant \boldsymbol{B}$ means $\boldsymbol{B}-\boldsymbol{A}$ is positive semi-definite. In this section, it will be shown that the window method subject to self-consistency delivers the augmented ordering relationship

$$
\overline{\boldsymbol{K}}_{\mathrm{UF}}^{(0)} \leqslant \overline{\boldsymbol{K}}_{\mathrm{UF}} \leqslant \overline{\boldsymbol{K}}_{\mathrm{PR}} \leqslant \overline{\boldsymbol{K}}_{\mathrm{LT}} \leqslant \overline{\boldsymbol{K}}_{\mathrm{LT}}^{(0)} \text {. }
$$

Now, a signature of a statistically representative microstructural sample is that its response is independent of the boundary conditions imposed. Therefore, the gap between $\overline{\boldsymbol{K}}_{\mathrm{UF}}^{(0)}$ and $\overline{\boldsymbol{K}}_{\mathrm{UF}}^{(0)}$ is an indication of how well the sample represents the desired homogenized response. Increasing sample size typically results in a diminishing gap. When the gap is sufficiently small, the sample is deemed representative for computational purposes. Since it is undesirable to use very large samples, the practical significance of the augmented ordering relationship becomes apparent. The window method delivers results that always fall between the two alternative $\mathrm{BC}$ results and therefore, similar to periodic BCs, one expects it to deliver faster convergence with increasing sample size. In this sense, the window method provides a tighter control over the sample size such that it is eventually possible to use a smaller sample compared to the situation without a frame, assuming LT- or UF-BCs are of concern. Where $\overline{\boldsymbol{K}}_{\mathrm{PR}}^{(0)}$ falls in (3.2) will be commented upon.

\subsection{Linear temperature boundary conditions}

To prove $\overline{\boldsymbol{K}}_{\mathrm{LT}} \leqslant \overline{\boldsymbol{K}}_{\mathrm{LT}}^{(0)}$, let $\mathbf{g}_{o}=\operatorname{grad}\left[\theta_{o}\right]$ and $\boldsymbol{q}_{o}$ constitute the solution to the problem without the frame under LT-BCs on $\partial \mathcal{V}$. The solution $\theta_{o}$ is extended into a fictitious frame domain via $\theta_{o}=\overline{\mathbf{g}} \cdot \boldsymbol{x}$ for $\boldsymbol{x} \in \mathcal{W}$. This extension is compatible with LT-BCs on $\partial \mathcal{V}$ or $\partial \mathcal{X}$. On the other hand, the solution with the window method using LT-BCs on $\partial \mathcal{X}$ is computed. The same frame material is employed in both cases. For the latter problem $\partial \mathcal{V}^{h}=\emptyset$ and, since the extended field is admissible, the minimum principle based on (2.1) implies

$$
-\frac{1}{2}|\mathcal{X}|\langle\mathbf{g} \cdot \mathbf{q}\rangle_{\mathcal{X}} \leqslant-\frac{1}{2}|\mathcal{X}|\left\langle\mathbf{g}_{o} \cdot \mathbf{q}_{o}\right\rangle_{\mathcal{X}},
$$


or, upon using (2.5),

$$
-\langle\mathbf{g}\rangle_{\mathcal{X}} \cdot\langle\boldsymbol{q}\rangle_{\mathcal{X}} \leqslant-\left\langle\mathbf{g}_{0}\right\rangle_{\mathcal{X}} \cdot\left\langle\mathbf{q}_{o}\right\rangle_{\mathcal{X}}
$$

By construction, $\langle\mathbf{g}\rangle_{\mathcal{X}}=\left\langle\mathbf{g}_{0}\right\rangle_{\mathcal{X}}=\overline{\mathbf{g}}$. Therefore, using (2.7), further manipulation yields

$$
-\overline{\mathbf{g}} \cdot\left(f_{v}\langle\boldsymbol{q}\rangle_{\mathcal{V}}+f_{w}\langle\boldsymbol{q}\rangle_{\mathcal{W}}\right) \leqslant-\overline{\mathbf{g}} \cdot\left(f_{v}\left\langle\boldsymbol{q}_{o}\right\rangle_{\mathcal{V}}+f_{w}\left\langle\boldsymbol{q}_{o}\right\rangle_{\mathcal{W}}\right),
$$

or

$$
\overline{\mathbf{g}} \cdot\left(f_{v} \overline{\mathbf{K}}_{\mathrm{LT}}\langle\mathbf{g}\rangle_{\mathcal{V}}+f_{w} \boldsymbol{K}_{w}\langle\mathbf{g}\rangle_{\mathcal{W}}\right) \leqslant \overline{\mathbf{g}} \cdot\left(f_{v} \overline{\boldsymbol{K}}_{\mathrm{LT}}^{(0)} \overline{\boldsymbol{g}}+f_{w} \boldsymbol{K}_{w} \overline{\mathbf{g}}\right) .
$$

Self-consistency is now invoked by replacing $\boldsymbol{K}_{w}$ by $\overline{\boldsymbol{K}}_{\mathrm{LT}}$ :

$$
\overline{\mathbf{g}} \cdot \overline{\mathbf{K}}_{\mathrm{LT}} \overline{\mathbf{g}} \leqslant \overline{\mathbf{g}} \cdot\left(f_{v} \overline{\boldsymbol{K}}_{\mathrm{LT}}^{(0)} \overline{\mathbf{g}}+f_{w} \overline{\boldsymbol{K}}_{\mathrm{LT}} \overline{\mathbf{g}}\right),
$$

or

$$
f_{v} \overline{\mathbf{g}} \cdot \overline{\mathbf{K}}_{\mathrm{LT}} \overline{\mathbf{g}} \leqslant f_{v} \overline{\mathbf{g}} \cdot \overline{\boldsymbol{K}}_{\mathrm{LT}}^{(0)} \overline{\mathbf{g}} .
$$

Since $\overline{\boldsymbol{g}}$ is arbitrary, this result states the positive semi-definiteness of $\overline{\boldsymbol{K}}_{\mathrm{LT}}^{(0)}-\overline{\boldsymbol{K}}_{\mathrm{LT}}$.

\subsection{Uniform flux boundary conditions}

The proof of $\overline{\boldsymbol{K}}_{\mathrm{UF}}^{(0)} \leqslant \overline{\boldsymbol{K}}_{\mathrm{UF}}$ closely follows the steps of the previous section. Only the beginning is stated. Let $\mathbf{g}_{o}=\operatorname{grad}\left[\theta_{o}\right]$ and $\boldsymbol{q}_{o}$ constitute the solution to the problem without the frame under UF-BCs on $\partial \mathcal{V}$. The solution $\boldsymbol{q}_{o}$ is extended into a fictitious frame domain via $\boldsymbol{q}_{o}=\overline{\boldsymbol{q}}$ for $\boldsymbol{x} \in \mathcal{W}$. This extension is compatible with UF-BCs on $\partial \mathcal{V}$ or $\partial \mathcal{X}$. On the other hand, the solution with the window method using UF-BCs on $\partial \mathcal{X}$ is computed. For the latter problem $\partial \mathcal{V}^{\theta}=\emptyset$ and, since the extended field is admissible, the complementary minimum principle based on (2.2) implies

$$
-\frac{1}{2}|\mathcal{X}|\langle\mathbf{q} \cdot \mathbf{g}\rangle_{\mathcal{X}} \leqslant-\frac{1}{2}|\mathcal{X}|\left\langle\mathbf{q}_{o} \cdot \mathbf{g}_{0}\right\rangle_{\mathcal{X}}
$$

The remaining steps are identical, leading to the positive semi-definiteness of $\overline{\boldsymbol{R}}_{\mathrm{LT}}^{(0)}-\overline{\boldsymbol{R}}_{\mathrm{LT}}$.

\subsection{Completing the ordering}

The end inequalities in (3.2) have been proven. To complete the ordering, it is sufficient to adapt the steps leading to the classical result (3.1). Only the idea in Hazanov and Huet (1994) is recalled here. Let $\left\{\theta_{\mathrm{LT}}, \theta_{\mathrm{UF}}, \theta_{\mathrm{PR}}\right\}$ be the three solutions under the three different boundary conditions using the window method, with corresponding flux and gradient fields. Moreover, it is assumed that $\overline{\boldsymbol{q}}$ in UF-BCs is chosen such that the average temperature gradient is enforced, i.e. $\langle\mathbf{g}\rangle_{\mathcal{X}}=\overline{\mathbf{g}}$. One observes:

1. $\boldsymbol{q}_{\mathrm{PR}}$ is an admissible flux field for the LT-BC problem. The complementary minimum principle is applicable, which leads to $\overline{\mathbf{K}}_{\mathrm{PR}} \leqslant \overline{\boldsymbol{K}}_{\mathrm{LT}}$.

2. $\theta_{\mathrm{PR}}$ is an admissible temperature field for the UF-BC problem. The minimum principle is applicable, which leads to $\overline{\boldsymbol{K}}_{\mathrm{UF}} \leqslant \overline{\boldsymbol{K}}_{\mathrm{PR}}$.

Again, invoking self-consistency is critical to completing the proofs.

It is noted that none of the arguments apply to $\overline{\boldsymbol{K}}_{\mathrm{PR}}^{(0)}$, i.e. where it stands in (3.2) cannot be predetermined. Regarding the limit response with increasing sample size, let $\boldsymbol{K}^{*}$ denote the effective thermal conductivity tensor that is obtained from a statistically representative sample. Arguments (Huet, 1990; Zohdi, 2002), based on the partitioning of a very large sample into smaller ones indicate that, provided ensemble averaging $\langle\langle\bullet\rangle\rangle$ of the arithmetic type is used,

$$
\left\langle\left\langle\overline{\boldsymbol{R}}_{\mathrm{UF}}^{(0)}\right\rangle\right\rangle^{-1} \leqslant \boldsymbol{K}^{*} \leqslant\left\langle\left\langle\overline{\boldsymbol{K}}_{\mathrm{LT}}^{(0)}\right\rangle\right\rangle .
$$

In other words, LT- and UF-BCs offer bounds on the unknown effective response. While the above bounds monitor only the mean response of the sample population, ordering relations for higher-order statistical measures may also be constructed (Zohdi, 2005). Moreover, these bounds can be argued to be arbitrarily refinable, i.e. the gap between them diminishes with increasing sample size. Presently, one cannot show that $\overline{\boldsymbol{K}}_{\mathrm{UF}}$ and $\overline{\boldsymbol{K}}_{\mathrm{LT}}$, which are obtained with the window method, constitute similar arbitrarily refinable bounds-their positions with respect to $\boldsymbol{K}^{*}$ are unknown. In this sense, the window method resembles periodic boundary conditions. This observation will be verified numerically.

\subsection{Choosing the frame width}

The width of the frame appears to be a free variable and this was of concern in the original work of Babuška et al. (1999) as well. Numerically, it is expected that choosing the frame width is similar to choosing the sample size-for a given sample size, one observes that $\overline{\boldsymbol{K}}$ saturates to a limit with increasing frame width. This expectation is based on the well-known 


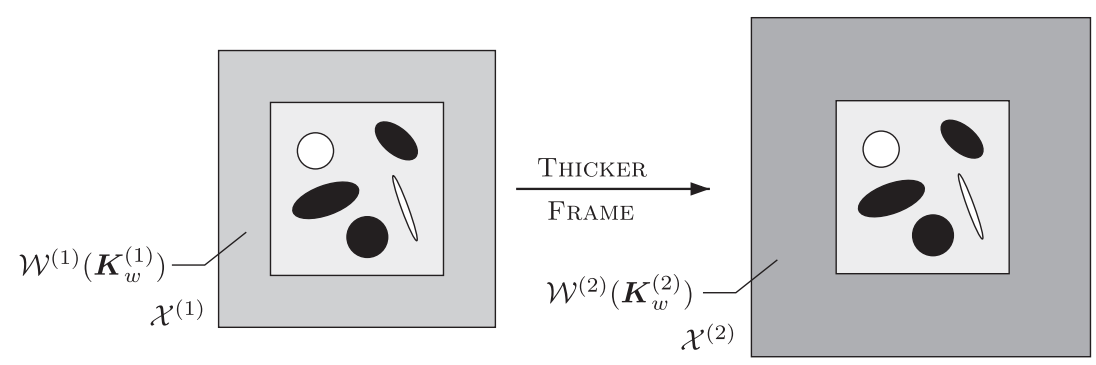

Fig. 2. Increasing frame thickness is depicted.

self-consistent scheme, where the frame is essentially an infinite surrounding medium, which delivers a unique response independent of whether LT- or UF-BCs are employed (Nemat-Nasser \& Hori, 1999). In fact, one can show that the augmented ordering complemented by increasing frame width delivers a monotonic closure of the gap between LT- and UF-BC results:

$$
\overline{\boldsymbol{K}}_{\mathrm{UF}}^{(0)} \leqslant \overline{\boldsymbol{K}}_{\mathrm{UF}}^{(1)} \leqslant \overline{\boldsymbol{K}}_{\mathrm{UF}}^{(2)} \leqslant \ldots \leqslant \overline{\boldsymbol{K}}_{w} \leqslant \ldots \leqslant \overline{\boldsymbol{K}}_{\mathrm{LT}}^{(2)} \leqslant \overline{\boldsymbol{K}}_{\mathrm{LT}}^{(1)} \leqslant \overline{\boldsymbol{K}}_{\mathrm{LT}}^{(0)} .
$$

Here, increasing superscript indicates the results obtained under increasing frame width and $\overline{\boldsymbol{K}}_{w}$ is the limit of the window method which corresponds to the classical self-consistent scheme. The proof is very similar to the one outlined for (3.2) therefore only the case for LT-BCs is discussed. Consider the setup in Fig. 2 and let $\theta_{o}$ constitute the solution to the problem with the thinner frame. It is extended into a fictitious frame domain $\mathcal{X}^{(2)} \backslash \mathcal{X}^{(1)}$ via $\theta_{o}=\overline{\mathbf{g}} \cdot \boldsymbol{x}$ and compared with the solution $\theta$ using the frame $\mathcal{W}^{(2)}$. Invoking self-consistency for both cases, the result (3.7) now takes the form

$$
\overline{\mathbf{g}} \cdot \overline{\boldsymbol{K}}_{\mathrm{LT}}^{(2)} \overline{\mathbf{g}} \leqslant \overline{\mathbf{g}} \cdot\left(f_{a} \overline{\boldsymbol{K}}_{\mathrm{LT}}^{(1)} \overline{\mathbf{g}}+f_{b} \overline{\boldsymbol{K}}_{\mathrm{LT}}^{(2)} \overline{\mathbf{g}}\right),
$$

where $f_{a}=\left|\mathcal{X}^{(1)}\right| /\left|\mathcal{X}^{(2)}\right|=1-f_{b}$, which indicates $\overline{\boldsymbol{K}}_{\mathrm{LT}}^{(2)} \leqslant \overline{\boldsymbol{K}}_{\mathrm{LT}}^{(1)}$.

Where the PR-BC results fall with respect to the limit $\overline{\boldsymbol{K}}_{w}$ is unknown at small frame widths. However, the limit obtained is independent of the BCs employed since PR-BC results are bounded in (3.2). On the other hand, this limit is not the effective limit $\boldsymbol{K}^{*}$ since the sample is not necessarily statistically representative. On the computational side, of course, it is undesirable to choose a very large width since this increases the size of the problem. Therefore, a trade-off exists between increasing the frame width and the sample size in practice.

\section{Numerical investigations}

In this section, the observations of the earlier discussions are briefly demonstrated through computations on two types of three-dimensional microstructures: (i) periodic microstructures where a unit cell corresponds to a sphere embedded in a matrix, and (ii) random microstructures which are obtained through the computed tomography scan (CT-scan) of hardened cement paste.

\subsection{Periodic media}

For investigations in the periodic setting, the unit cell width is set to 10 units while the diameter of the sphere is 9 units, corresponding to a volume fraction of approximately 0.38 . The thermal conductivity of the sphere is $500 \mathrm{~W} / \mathrm{mK}$ and the one of the matrix is $1 \mathrm{~W} / \mathrm{mK}$. The unit-cell displays macroscopic isotropy, represented by $\bar{k}=\left\|\langle\boldsymbol{q}\rangle_{\mathcal{V}}\right\| /\left\|\langle\boldsymbol{g}\rangle_{\mathcal{V}}\right\|$. The unit cell is
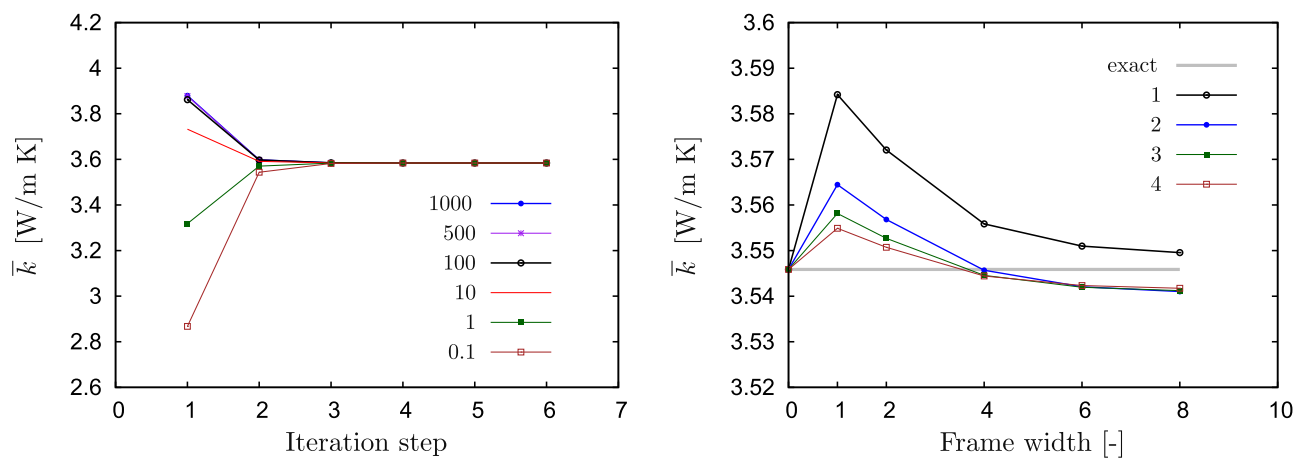

Fig. 3. (Left) Iterations under PR-BCs with respect to various initial thermal conductivities $k$ [W/mK] assigned to the frame of 1 unit around a unit cell. (Right) Influence of the frame width and number of unit cells per direction on the macroscopic thermal conductivity under PR-BCs. Self-consistency is ensured. 

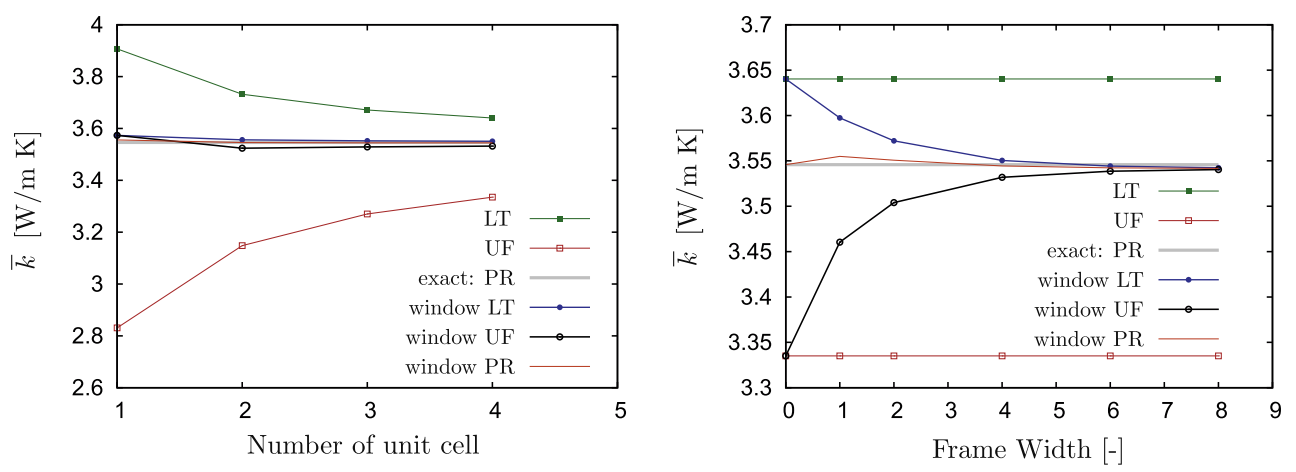

Fig. 4. (Left) Comparison of the macroscopic response with and without a frame (fixed width at 1 unit) under different BCs and increasing number of unit cells per spatial direction of the sample. (Right) The influence of the frame width under different BCs with four unit cells per direction.
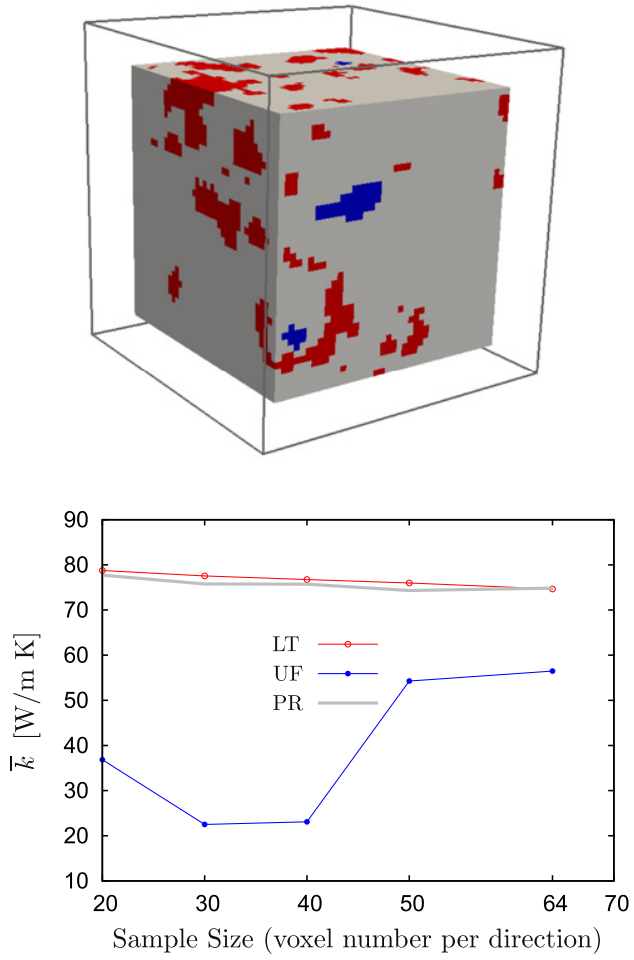
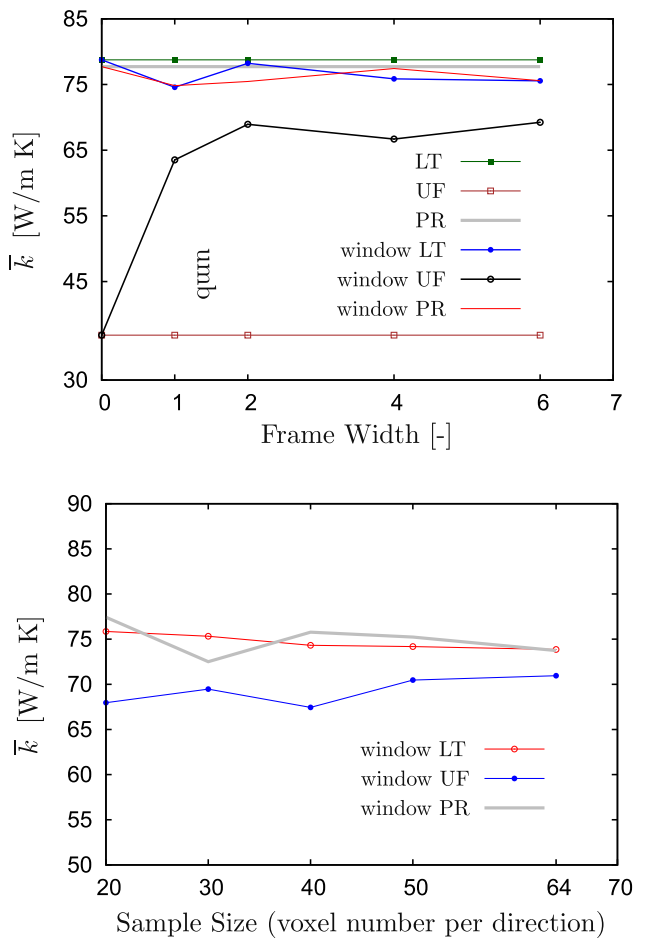

Fig. 5. (Top-left) The CT-scan of an HCP sample with 64 voxels per direction, embedded in a frame. (Top-right) effect of the frame width on a single sample with 20 voxels per direction subjected to different BCs. (Bottom-Left) Using a frame width of 4 units, the effect of the sample size is demonstrated without a frame. 150 samples are tested and the results are averaged to alleviate randomness effects. (Bottom-Right) The same set of samples are tested with a frame.

embedded within a homogeneous frame and various BCs are directly prescribed on the frame to investigate the influence of the window method on the macroscopic response. The number of self-consistency iterations vary depending on the conductivity initially assigned to the frame material but is typically less than four as summarized in Fig. 3. The influence of the width of the frame is also illustrated in Fig. 3 based on PR-BCs, where it is observed that the results obtained with a frame converge to a limit with increasing frame width. In this periodic setting, it is also observed that this limit is very close to the exact result that is obtained with PR-BCs directly on the unit cell. Clearly, for a given frame width, increasing the number of unit cells in the periodic sample also drives the results closer to the exact result as expected, which is also shown in Fig. 4 for the three types of BCs without a frame as well as with a fixed frame width of 4 units. Finally, the results (3.2) and (3.11) are also demonstrated in Fig. 4, where strict ordering among different BCs as well as their convergence to a common limit with increasing frame width are observed. 


\subsection{Random media}

As an application to random media, hardened cement paste (HCP) is considered. The microstructure originates from a micro-CT scan although the assigned conductivities are artificial. The representation of an HCP sample embedded in a frame is illustrated in Fig. 5 where the hydration products are indicated with the gray color, the unhydrated residual klinker with blue and the micropores with red. The volume fraction of the klinker is 0.84 , the one of unhydrated residual is 0.02 and the one of micropores is 0.14 . The thermal conductivity of the klinker is set to $100 \mathrm{~W} / \mathrm{mK}$ while the others are set to $1 \mathrm{~W} / \mathrm{mK}$. In presenting the results, a single quantity $\bar{k}$ is monitored assuming macroscopic isotropy. This assumption is only satisfied for sufficiently large samples and therefore leads to some deviation from the predicted ordering relations for smaller sample sizes, the size referring to the number of voxels per spatial direction of the sample. This is observed in Fig. 5 on a sample with 20 voxels per direction. Nevertheless, the response under different BCs approach each other with increasing frame width. In order to obtain a statistically representative sample, larger CT-scans have to be employed. To alleviate randomness effects, 150 samples are tested per sample size. If no frame is used, it is observed that the LT- and PR-BC results are close to each other but they remain significantly far away from UF-BC predictions. This large gap casts a doubt on the quality of the macroscopic predictions since the types of BCs should not affect the response of a statistically representative sample. When the same computations are carried out with a window, it is observed that the gap is small even at small sample sizes. Consequently, one can state with confidence that the macroscopic conductivity is in the range of 70 to $75 \mathrm{~W} / \mathrm{mK}$. Considering that the frame width is only 4 units and that only two iterations were sufficient to ensure self-consistency, the additional cost of using the window method is negligible with this observed advantage. This advantage translates into a tighter control over the sample size when analyzing randomly heterogeneous media.

\section{Conclusion}

The observed optimal convergence behavior of the homogenization results with the window method has been proven analytically and demonstrated numerically with periodic particulate and random digital microstructures. In contrast to the three types of classical boundary conditions, it was shown that the window method delivers a unique macroscopic response for any given sample size independent of the type of boundary conditions imposed-provided that the window is chosen to be sufficiently thick and that the overall scheme satisfies self-consistency. For randomly heterogeneous media, the window method enables a tighter control over the sample size in trying to ensure statistical representativeness. Although the presentation was pursued in a thermal context, the underlying variational basis is applicable to linear elasticity in a straightforward manner. Specifically, denoting the macroscopic fourth-order stiffness tensor by $\overline{\boldsymbol{I E}}$, the counterpart of the ordering relation (3.2) which delivers refined predictions reads

$$
\overline{\boldsymbol{I E}}_{\mathrm{UF}}^{(0)} \leqslant \overline{\boldsymbol{I E}}_{\mathrm{UF}} \leqslant \overline{\boldsymbol{I E}}_{\mathrm{PR}} \leqslant \overline{\boldsymbol{I E}}_{\mathrm{LT}} \leqslant \overline{\boldsymbol{I E}}_{\mathrm{LT}}^{(0)}
$$

while the monotonic convergence relation (3.11) of the window method predictions to a common limit $\overline{\boldsymbol{I E}}_{w}$ as the frame width is increased under different BCs may be stated as

$$
\overline{\boldsymbol{I E}}_{\mathrm{UF}}^{(0)} \leqslant \overline{\boldsymbol{I E}}_{\mathrm{UF}}^{(1)} \leqslant \overline{\boldsymbol{I E}}_{\mathrm{UF}}^{(2)} \leqslant \ldots \leqslant \overline{\boldsymbol{I E}}_{w} \leqslant \ldots \leqslant \overline{\boldsymbol{I E}}_{\mathrm{LT}}^{(2)} \leqslant \overline{\boldsymbol{I}}_{\mathrm{LT}}^{(1)} \leqslant \overline{\boldsymbol{I E}}_{\mathrm{LT}}^{(0)} .
$$

\section{References}

Babuška, I., Andersson, B., Smith, P. J., \& Levin, K. (1999). Damage analysis of fiber composites - Part I. Statistical analysis on fiber scale. Computer Methods in Applied Mechanics and Engineering, 172, 27-77.

Benveniste, Y. (2008). Revisiting the generalized self-consistent scheme in composites: Clarification of some aspects and a new formulation. Journal of the Mechanics and Physics of Solids, 56, 2984-3002.

Benveniste, Y., \& Milton, G. W. (2011). An effective medium theory for multi-phase matrix-based dielectric composites with randomly oriented ellipsoidal inclusions. International Journal of Engineering Science, 49, 2-16.

Düster, A., Sehlhorst, H-G., \& Rank, E. (2012). Numerical homogenization of heterogeneous and cellular materials utilizing the finite cell method. Computational Mechanics, 50, 413-431.

Hain, M., \& Wriggers, P. (2008). Numerical homogenization of hardened cement paste. Computuational Mechanics, 42, $197-212$.

Hazanov, S., \& Huet, C. (1994). Order relationships for boundary conditions effect in heterogeneous bodies smaller than the representative volume. Journal of the Mechanics and Physics of Solids, 42, 1995-2011.

Huet, C. (1990). Application of variational concepts to size effects in elastic heterogeneous bodies. Journal of the Mechanics and Physics of Solids, 38, $813-841$.

Jiang, B., \& Weng, G. J. (2004). A generalized self-consistent polycrystal model for the yield strength of nanocrystalline materials. Journal of the Mechanics and Physics of Solids, 52, 1125-1149.

Kanaun, S. K., \& Levi, V. M. (2008). Self-Consistent Methods for Composites-Vol. 1: Static Problems. Static problems. Self-consistent methods for composites (Vol. 1). Springer.

Krabbenhøft, K., Hain, M., \& Wriggers, P. (2008). Computation of effective cement paste diffusivities from microtomographic images. In V. Kompis (Ed.), Composites with micro- and nano-structure: Computational modeling and experiments (pp. 281-297). Berlin, Heidelberg, New York: Springer.

Miehe, C., \& Koch, A. (2002). Computational micro-to-macro transitions of discretized microstructures undergoing small strains. Archive of Applied Mechanics, 72, 300-317.

Nemat-Nasser, S., \& Hori, M. (1999). Micromechanics: Overall properties of heterogeneous materials (2nd ed.). Amsterdam: North-Holland.

Ostoja-Starzewski, M. (2006). Material spatial randomness: From statistical to representative volume element. Probabilistic Engineering Mechanics, 21, $112-132$.

Pavliotis, G. A., \& Stuart, A. M. (2008). Multiscale methods: Averaging and homogenization. Berlin, Heidelberg, New York: Springer. 
Su, Y., \& Weng, G. J. (2006). A self-consistent polycrystal model for the spontaneous polarization of ferrorelectric ceramics. Proceedings of the Royal Society A, $462,1763-1789$.

Torquato, S. (2002). Random heterogeneous materials: Microstructure and macroscopic properties. Berlin, Heidelberg, New York: Springer.

Zohdi, T. I. (2002). Bounding envelopes in multiphase material design. Journal of Elasticity, 66, 47-62.

Zohdi, T. I. (2005). Statistical ensemble error bounds for homogenized microheterogeneous solids. Zeitschrift für angewandte Mathematik und Physik, 56, 497-515.

Zohdi, T. I. (2010). Simulation of coupled microscale multiphysical-fields in particulate-doped dielectrics with staggered adaptive FDTD. Computer Methods in Applied Mechanics and Engineering, 199, 3250-3269.

Zohdi, T. I., \& Wriggers, P. (2005). Introduction to computational micromechanics. Berlin, Heidelberg, New York: Springer. 